\title{
Surfactant Attenuates Air Embolism-Induced Lung Injury by Suppressing NKCC1 Expression and NF-кB Activation
}

\author{
Chou-Chin Lan, ${ }^{1,2}$ Yao-Kuang Wu, ${ }^{1,2}$ Chung-Kan Peng, ${ }^{3,4}$ Kun-Lun Huang, ${ }^{3,4}$ and \\ Chin-Pyng $\mathrm{Wu}^{5,6}$
}

\begin{abstract}
Excessive amounts of air can enter the lungs and cause air embolism (AE)-induced acute lung injury (ALI). Pulmonary AE can occur during diving, aviation, and iatrogenic invasive procedures. AE-induced lung injury presents with severe hypoxia, pulmonary hypertension, microvascular hyper-permeability, and severe inflammatory responses. Pulmonary AEinduced ALI is a serious complication resulting in significant morbidity and mortality. Surfactant is abundant in the lungs and its function is to lower surface tension. Earlier studies have explored the beneficial effects of surfactant in ALI; however, none have investigated the role of surfactant in pulmonary AE-induced ALI. Therefore, we conducted this study to determine the effects of surfactant in pulmonary AE-induced ALI. Isolated-perfused rat lungs were used as a model of pulmonary $\mathrm{AE}$. The animals were divided into four groups ( $n=6$ per group): sham, air embolism (AE), $\mathrm{AE}+\operatorname{surfactant}(0.5 \mathrm{mg} / \mathrm{kg})$, and $\mathrm{AE}+$ surfactant $(1 \mathrm{mg} / \mathrm{kg})$. Surfactant pretreatment was administered before the induction of pulmonary AE. Pulmonary AE was induced by the infusion of $0.7 \mathrm{cc}$ air through a pulmonary artery catheter. After induction of air, pulmonary AE was presented with pulmonary edema, pulmonary microvascular hyper-permeability, and lung inflammation with neutrophilic sequestration. Activation of NF-KB was observed, along with increased expression of pro-inflammatory cytokines, and $\mathrm{Na}-\mathrm{K}-\mathrm{Cl}$ cotransporter isoform 1 (NKCC1). Surfactant suppressed the activation of NF- $\mathrm{KB}$ and decreased the expression of proinflammatory cytokines and $\mathrm{NKCC} 1$, thereby attenuating AE-induced lung injury. Therefore, AE-induced ALI presented with pulmonary edema, microvascular hyper-permeability, and lung inflammation. Surfactant suppressed the expressions of NF- $\mathrm{BB}$, pro-inflammatory cytokines, and $\mathrm{NKCC} 1$, thereby attenuating AE-induced lung injury.
\end{abstract}

KEY WORDS: air embolism; Na-K-Cl cotransporter isoform 1; lung injury; surfactant.

Drs. Kun-Lun Huang and Chin-Pyng Wu contributed equally to this work and are co-corresponding authors

\footnotetext{
${ }^{1}$ Division of Pulmonary Medicine, Taipei Tzu Chi Hospital, Buddhist Tzu Chi Medical Foundation, New Taipei City, Taiwan

${ }^{2}$ School of Medicine, Tzu-Chi University, Hualien, Taiwan

${ }^{3}$ Division of Pulmonary Medicine, Tri-Service General Hospital, Taipei, Taiwan
}

\footnotetext{
${ }^{4}$ Institute of Undersea and Hyperbaric Medicine, National Defense Medical Center, Taipei, Taiwan

${ }^{5}$ Department of Critical Care Medicine, Landseed International Hospital, Tao-Yuan, Taiwan

${ }^{6}$ To whom correspondence should be addressed at Department of Critical Care Medicine, Landseed International Hospital, Tao-Yuan, Taiwan. Email: wucp@landseed.com.tw
} 


\section{INTRODUCTION}

Pulmonary air embolism (AE) occurs when air enters the circulatory system and lungs [4]. There are a lot of iatrogenic invasive procedures that can lead to pulmonary $\mathrm{AE}$, including radical neck dissection, laparoscopic surgery, therapeutic bronchoscopy, central venous access, and hemodialysis catheters. [4, 7]. Pulmonary AE is also known to occur in pilots during high-altitude flights and divers undergoing decompression after hyperbaric exposure.

Under normal conditions, small amounts of air are usually absorbed from the pulmonary vasculature. However, when the amounts of air exceed the absorption capability of the lung, acute lung injury (ALI) can result and is termed AE-induced ALI [19]. The AE-induced ALI presents with pulmonary edema, pulmonary hypertension, impaired gas exchange, and profound hypoxia [7]. AEinduced ALI is therefore a serious complication resulting in significant morbidity and mortality [4, 7].

The mechanisms of AE-induced ALI are multiple factors $[12,14,22]$. Pulmonary AE causes physical obstruction of microvasculature, leads to permeability changes, and releases inflammatory mediators. Air bubbles irritate the pulmonary endothelium and induce a series of inflammatory responses [14]. Neutrophils also play an important role in the pathogenesis of AE-induced ALI [22] by interacting with the air emboli and activating inflammatory responses, causing the release of reactive oxygen species, proteases, and cytokines and up-regulate the production of adhesion molecules and result in further lung damage [12]. Together, these factors lead to lung injury with vascular hyper-permeability, pulmonary ede$\mathrm{ma}$, and poor gas exchanges [12, 14, 22].

Surfactant is abundant in the lungs. It alters respiratory mechanics by its surface-active properties, thereby lowering surface tension. Surfactant dysfunction contributes to the disturbed physiology in ALI with progressive loss of aerated volume and exacerbation of ventilation/perfusion mismatch [6]. Both animal and human studies suggested that surfactant therapy is effective for ALI and acute respiratory distress syndrome (ARDS) [2, 15, 25]. Amigoni et al. showed that surfactant is effective in children and infants with ARDS [2]. Nakajima et al. suggested that surfactant replacement reduced the levels of inflammatory cytokines and secretory phospholipase $\mathrm{A}_{2}$ in pigs with gastric acid aspiration-induced lung injury [15]. While exogenous surfactant administration seems to improve lung function in ALI [25], the effects of surfactant in AEinduced ALI are still unclear.
Alveolar fluid regulation plays a critical role in the development of pulmonary edema in lung injury. The pulmonary ion channel $\mathrm{Na}-\mathrm{K}-\mathrm{Cl}$ cotransporter isoform 1 (NKCC1) is important in alveolar fluid regulation. NKCC1 locates basolaterally in the lung epithelium that mediates a net influx of ions into alveolar cells [1]. The chemical gradient that creates the driving force for water transport is therefore mediated primarily by $\mathrm{NKCC} 1$ [1]. Besides, NKCC1 is known to regulate lung inflammation [16, 24]. One previous study showed that mice lacking NKCC1 are protected from bacterial pneumonia [16]. Previously, we also suggested that $\mathrm{NKCC} 1$ plays an important role in IRinduced lung injury, in that increased expression of NKCC1 leads to more severe ALI. In the current study, we further investigated the role of $\mathrm{NKCCl}$ in $\mathrm{AE}$-induced lung injury.

Surfactant and NKCC1 are both important in the regulation of lung water and inflammation. We hypothesized that the administration of surfactant should be a benefit for AE-induced lung injury. However, studies exploring surfactant and $\mathrm{NKCC1}$ in AE-induced lung injury are lacking. Therefore, this study aimed to determine the effects of surfactant and changes in NKCC1 in AE-induced ALI.

\section{MATERIALS AND METHODS}

\section{Isolation and Perfusion of the Lungs}

The National Science Council and Animal Review Committee of the National Defense Medical Center (Taipei, Taiwan) approved the protocol for this study. The animals were cared for in accordance with the "Guide for the Care and Use of Laboratory Animals" published by the US National Institutes of Health.

Procedures regarding the preparation of isolatedperfused lungs in situ in the chest were as previously described [11]. Male Sprague-Dawley rats were anesthetized through intraperitoneal injection of pentobarbital sodium $(50 \mathrm{mg} / \mathrm{kg})$. After confirmation of deep anesthesia, tracheostomy was performed and a cannula was inserted into the trachea. The lungs were ventilated with a humidified gas mixture containing $5 \% \mathrm{CO}_{2}$ in air at a frequency of $60 \mathrm{cycle} / \mathrm{min}$, tidal volume of $3 \mathrm{~mL}$, and end-expiratory pressure of $1 \mathrm{~cm} \mathrm{H}_{2} \mathrm{O}$. Median sternotomy was performed and heparin $(1 \mathrm{U} / \mathrm{g}$ of body weight) was injected into the right ventricle.

A peristaltic pump (Model 1203, Harvard Apparatus) was used to perfuse the lungs with re-circulated perfusate 
comprising blood mixed with physiological salt solution (119 mM NaCl, $4.7 \mathrm{mM} \mathrm{KCl}, 1.17 \mathrm{mM} \mathrm{MgSO}_{4}, 22.6 \mathrm{mM}$ $\mathrm{NaHCO}_{3}, 1.18 \mathrm{mM} \mathrm{KH} \mathrm{PO}_{4}, 1.6 \mathrm{mM} \mathrm{CaCl}, 5.5 \mathrm{mM}$ glucose, and $50 \mathrm{mM}$ sucrose). Bovine albumin (4 g/dL) was added to maintain the osmolarity of the perfusate. A cannula was placed in the left atrium via the left ventricle to collect the effluent perfusate for re-circulation.

The perfusion rate and temperature were maintained at $8-10 \mathrm{~mL} / \mathrm{min}$ (by a roller pump) and $37^{\circ} \mathrm{C}$ (by a water bath), respectively. The preparation was placed on an electronic balance with the isolated lungs remaining in situ.

\section{Experimental Protocols and Induction of Air Embolism-Induced Acute Lung Injury}

The animals were divided into four groups ( $n=6$ per group): sham, AE, AE + surfactant $(0.5 \mathrm{mg} / \mathrm{kg})$, and $\mathrm{AE}+$ surfactant $(1 \mathrm{mg} / \mathrm{kg})$. Pretreatment surfactant was administered before induction of AE. Pulmonary AE was induced through infusion of $0.7 \mathrm{cc}$ air through the pulmonary artery catheter.

All groups were studied for pulmonary microvascular permeability (Kf), alveolar fluid clearance (AFC), lung histopathology, lung weight/body weight ratio (LW/BW), lung wet/dry weight ratio (W/D), and levels of tumor necrosis factor-alpha (TNF- $\alpha$ ), chemokine (C-X-C motif) ligand 1 (CXCL1), IL-1ß, nuclear factor-kappa B (NF-kB), inhibitor of NF-kB alpha (IкB- $\alpha)$, and NKCC1.

\section{Microvascular Permeability}

The measurement of $\mathrm{Kf}$ in isolated lungs was conducted as previously described [11]. An index of Kf was determined from the lung weight change induced by elevated pulmonary venous pressure (PVP). The PVP was rapidly elevated by $10 \mathrm{~cm} \mathrm{H}_{2} \mathrm{O}$ for $7 \mathrm{~min}$. The slow, steady phase of weight gain as a function of time $(\Delta \mathrm{W} /$ $\Delta \mathrm{T}$ ) was plotted on a semi-logarithmic paper and then extrapolated to zero time to obtain the initial rate of trans-capillary filtration. From this plot, Kf was defined as the $y$-intercept $(\mathrm{gm} / \mathrm{min})$ divided by PVP $(10 \mathrm{~cm}$ $\mathrm{H}_{2} \mathrm{O}$ ) and lung weight and expressed in whole units of grams per minute per centimeter of $\mathrm{H}_{2} \mathrm{O}$ multiplied by $100 \mathrm{~g}[28]$.

\section{Alveolar Fluid Clearance Measurement}

AFC was measured using an in situ lung model as previously described [13]. In brief, the euthanized rats were maintained at $37-38{ }^{\circ} \mathrm{C}$ using a heating pad, and the lungs were inflated with $100 \% \mathrm{O}_{2}$ at $7 \mathrm{cmH}_{2} \mathrm{O}$ continuous positive airway pressure throughout the experiment. Subsequently, $12.5 \mathrm{~mL} / \mathrm{kg}$ body weight of instillate containing fluorescein isothiocyanate (FITC)-conjugated albumin (Sigma-Aldrich, St. Louis, MO, USA) was delivered into the lungs over $1 \mathrm{~min}$. An alveolar fluid sample $(100 \mu \mathrm{L})$ was aspirated $1 \mathrm{~min}$ after instillation and $15 \mathrm{~min}$ later. The aspirates were centrifuged at $3000 \times g$ for $10 \mathrm{~min}$, and the fluorescence activity in the supernatant was measured in duplicate. AFC was computed from the increase in alveolar fluid albumin concentration using the equation:

$\mathrm{AFC}=(C f-C i) / C f \times 100$

where $C i$ and $C f$ represent the initial and final concentrations of FITC-albumin in the aspirate at 1 and $15 \mathrm{~min}$, as assessed by the fluorescence activity measurements.

\section{Pro-inflammatory Cytokines Levels in Perfusate}

The expression levels of pro-inflammatory cytokines including TNF- $\alpha$, CXCL1, and IL-1ß were determined by commercially available enzyme-linked immunosorbent assays (ELISA) (R\&D Systems Inc., Minneapolis, MN).

\section{Pulmonary Edema}

The lung W/D ratio was used as an indicator of pulmonary edema. After the experiment, part of the right middle lobe was weighed and then dried in an oven at $60{ }^{\circ} \mathrm{C}$ for $48 \mathrm{~h}$. The wet and dry weights were then measured to calculate the lung $\mathrm{W} / \mathrm{D}$ ratio.

\section{Lung Histopathology}

Histopathologic examination was performed to verify the micro-anatomic features of AE-induced ALI and to assess the effects of surfactant. After the experiment, the lungs were removed and fixed with $10 \%$ formaldehyde at $20 \mathrm{cmH}_{2} \mathrm{O}$ infused through the trachea. The tissues were immersed in $10 \%$ formaldehyde fixative for $24 \mathrm{~h}$, embedded in paraffin wax, and cut into 4-6 $\mu \mathrm{m}$-thick sections using a microtome. The sections were then stained with hematoxylin and eosin (H\&E) to assess interstitial edema, inflammation, and degree of neutrophilic infiltration.

\section{Tissue Neutrophil Quantification}

For tissue neutrophilic quantification, H\&Estained sections were used to count the number of neutrophils per high power field $(\times 400)$ [23]. For each 
slide, neutrophils were counted in 10 non-overlapping high power fields [9].

\section{Immuno-blotting}

Cytoplasmic and nuclear proteins were extracted from frozen lung tissues using the Nuclear/Cytosol Extraction kit (BioVision, Inc., Mountain View, CA). Protein concentrations were determined by BCA protein assays and equal amounts of lung homogenates ( $30 \mu \mathrm{g} / \mathrm{lane})$ were fractionated on 10-12\% sodium dodecyl sulfate-polyacrylamide gel electrophoresis (SDS-PAGE) gels and transferred to Hybond polyvinylidene fluoride membranes. The membranes were blocked by incubation in phosphate-buffered saline containing $0.1 \%$ Tween 20 and $5 \%$ non-fat milk for $1 \mathrm{~h}$ at room temperature.

The blots were incubated with antibodies against phosphorylated NF-kB p65, IкB- $\alpha$ and NKCC1 (Cell Signaling Technology, Danvers, MA) overnight at $4{ }^{\circ} \mathrm{C}$. The blots were then washed three times for 10 min using phosphate-buffered saline containing $0.1 \%$ Tween 20 . The blots were incubated with horseradish peroxidase linked anti-rabbit immunoglobulin $\mathrm{G}(1: 40,000)$ or anti-goat immunoglobulin $\mathrm{G}(1: 50,000)$ for $1 \mathrm{~h}$ at room temperature, and then washed three times in phosphate-buffered saline containing $0.1 \%$ Tween 20 for $10 \mathrm{~min}$. The bands were visualized using enhanced chemiluminescence reagents and by exposing the blot to a radiography film. The blots were then stripped and incubated with an anti-TATA antibody (for nuclear protein, diluted 1:1000; Abcam,
Cambridge, MA) or anti- $\beta$-actin antibody (for cytoplasmic protein, diluted 1:10,000; Sigma, St. Louis, MO) to ensure equal loading.

\section{Data Analysis}

All statistical analyses were performed using the SPSS software 18.0 (SPSS Inc., Chicago, IL). Differences between groups were evaluated using Kruskal-Wallis followed by post hoc comparisons with Games-Howell tests (intergroup comparison). Statistical significance was set at $p<0.05$.

\section{RESULTS}

\section{Surfactant Decreased Air Embolism-Induced Pulmo- nary Edema (Fig. 1)}

Both LW/BW ratio (Fig. 1a) and lung W/D ratio (Fig. 1b) were significantly increased in the AE group as compared to the sham group $(p<0.05)$. Surfactant $1 \mathrm{mg} / \mathrm{kg}$ significantly decreased AE-induced pulmonary edema as compared to the AE group $(p<0.05)$.

\section{Surfactant Decreased Air Embolism-Induced Micro- vascular Hyper-Permeability (Fig. 2)}

The Kfl at baseline was similar among these groups $(p>0.05)$ (Fig. 2a). The Kf2 was significantly increased in the AE group compared to the sham group $(p<0.05)$.
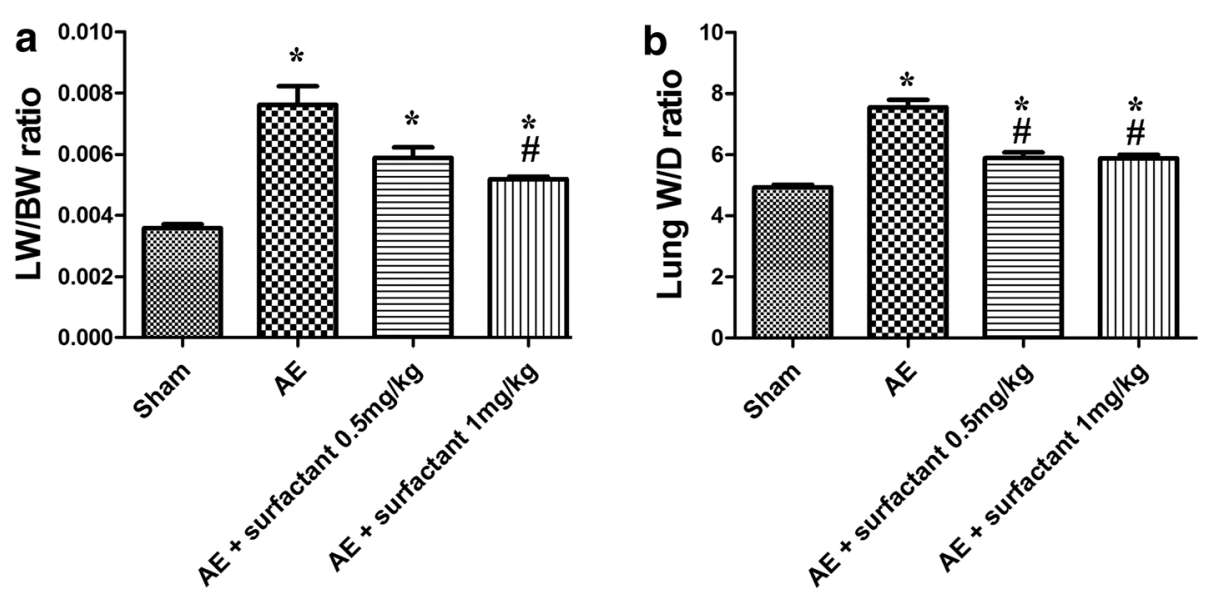

Fig. 1. Surfactant decreased air embolism-induced pulmonary edema. a Lung weight/body weight (LW/BW) and wet/dry weight ratio (W/D) were significantly increased in the air embolism (AE) group compared to the sham group $(p<0.05)$. Surfactant $1 \mathrm{mg} / \mathrm{kg}$ significantly decreased LW/BW and W/D compared to the AE group $(p<0.05)$. There was a significant difference between the *Sham $(p<0.05)$ and ${ }^{\#} \mathrm{AE}(p<0.05)$ groups. Abbreviations: $\mathrm{AE}$, air embolism; LW/BW, lung weight/body weight ratio; W/D: wet/dry weight ratio. 

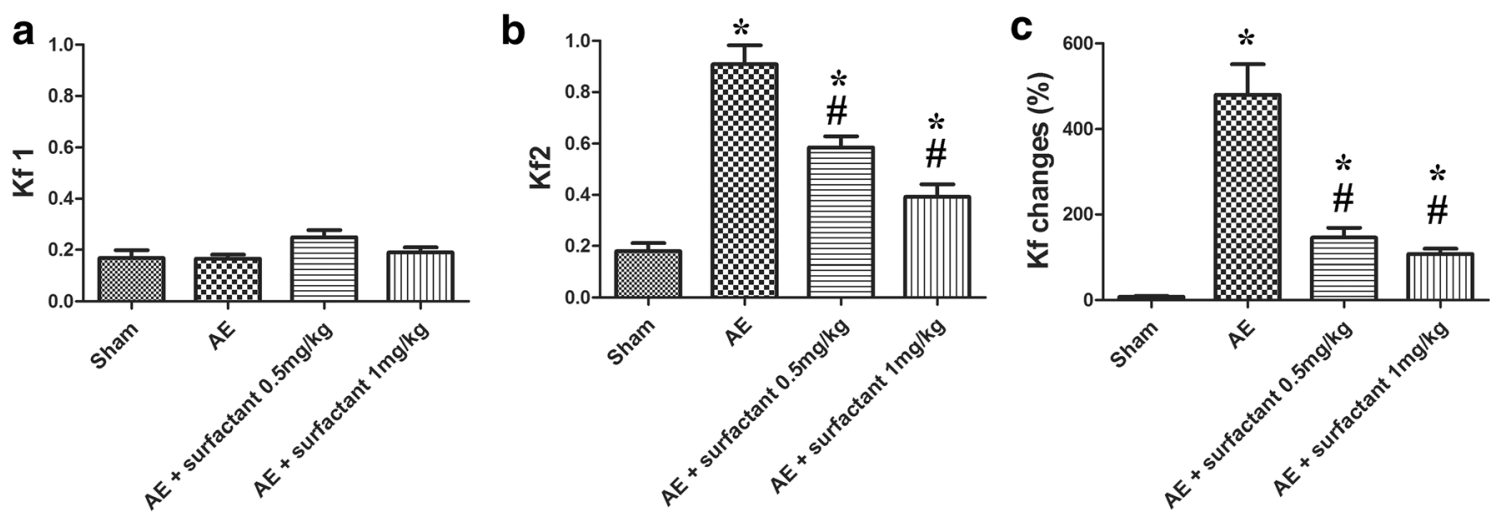

Fig. 2. Surfactant decreased air embolism-induced pulmonary microvascular hyper-permeability. a Pulmonary microvascular permeability at baseline (Kf1) was similar among these groups $(p>0.05$ ). b Pulmonary microvascular permeability after air embolism (Kf2) was significantly increased in the air embolism (AE) group. Surfactant $1 \mathrm{mg} / \mathrm{kg}$ significantly decreased post-AE microvascular permeability, compared to the AE group $(p<0.05)$. c Changes in pulmonary microvascular permeability (Kf) were prominent in the AE group and attenuated in rats with surfactant $1 \mathrm{mg} / \mathrm{kg}$ compared to the AE group $(p<0.05)$. There was a significant difference between the ${ }^{*}$ Sham $(p<0.05)$ and ${ }^{\#} \mathrm{AE}(p<0.05)$ groups. Abbreviations: $\mathrm{AE}$, air embolism; Kf1, pulmonary microvascular permeability at baseline; Kf2, pulmonary microvascular permeability after AE.

Surfactant $1 \mathrm{mg} / \mathrm{kg}$ significantly decreased post-AE microvascular permeability, compared to the AE group $(p<0.05)$. The changes of $\mathrm{Kf}$ between baseline and post-AE were more prominent in the $\mathrm{AE}$ group compared to the sham group $(p<0.05)$, and were attenuated in the rats treated with surfactant $1 \mathrm{mg} / \mathrm{kg}(p<0.05)$.

\section{Surfactant Restored Alveolar Fluid Clearance (Fig. 3)}

$\mathrm{AFC}$ is an indicator of water re-absorption from the alveolar space into the interstitium. AFC was significantly lower in the $\mathrm{AE}$ group compared to the sham group $(p<0.05)$, and surfactant $1 \mathrm{mg} / \mathrm{kg}$ significantly restored $\mathrm{AFC}(p<0.05$, compared with the AE group).

\section{Surfactant Attenuated Lung Injury (Fig. 4)}

The rats of the sham group had normal histology (Figs. 4a), while the AE group showed prominent neutrophilic sequestration and inter-alveolar septum thickening (Fig. 4b). Lung injury was less severe in rats receiving surfactant $0.5 \mathrm{mg} / \mathrm{kg}$ (Fig. 4c) and was markedly attenuated by surfactant $1 \mathrm{mg} / \mathrm{kg}$ (Fig. 4d).

\section{Surfactant Attenuated Lung Injury and Neutrophilic Sequestration (Fig. 5)}

Quantified neutrophilic counts showed significantly higher numbers of neutrophils in the AE group compared to the sham group $(p<0.05)$; the numbers were significantly reduced by the administration of surfactant $1 \mathrm{mg} / \mathrm{kg}(p<0.05)$.

\section{Surfactant Decreased Expressions of Pro- inflammatory Cytokines (Fig. 6)}

Expression levels of pro-inflammatory cytokines, IL$1 \beta$ (6a), TNF- $\alpha$ (Fig. 6b), and CXCL-1 (6c) were significantly increased in the AE group compared to the sham group $(p<0.05)$. Surfactant $1 \mathrm{mg} / \mathrm{kg}$ decreased the expressions of these cytokines after $\mathrm{AE}(p<0.05)$.

\section{Surfactant Decreased Air Embolism-Induced Expres- sion of NF-KB Activation and Nuclear Translocation} (Fig. 7)

In the AE group, the cytoplasmic level of phosphorylated NF-KB p65 (Fig. 7a) was increased, whereas that of IKB- $\alpha$ (Fig. 7b) was significantly suppressed as compared to the sham group $(p<0.05)$. Surfactant $1 \mathrm{mg} / \mathrm{kg}$ reduced phosphorylated NF- $\kappa$ B p 65 level and restored I $\kappa$ B- $\alpha$ level as compared to the $\mathrm{AE}$ group $(p<0.05)$.

\section{Surfactant Decreased Air Embolism-Induced Expres- sion of NKCC1 (Fig. 8)}

The expression of $\mathrm{NKCC} 1$ was significantly increased in the $\mathrm{AE}$ group as compared to the sham group $(p<0.05)$. Surfactant $1 \mathrm{mg} / \mathrm{kg}$ significantly decreased the expression of $\mathrm{NKCC} 1$, compared to the AE group $(p<0.05)$. 


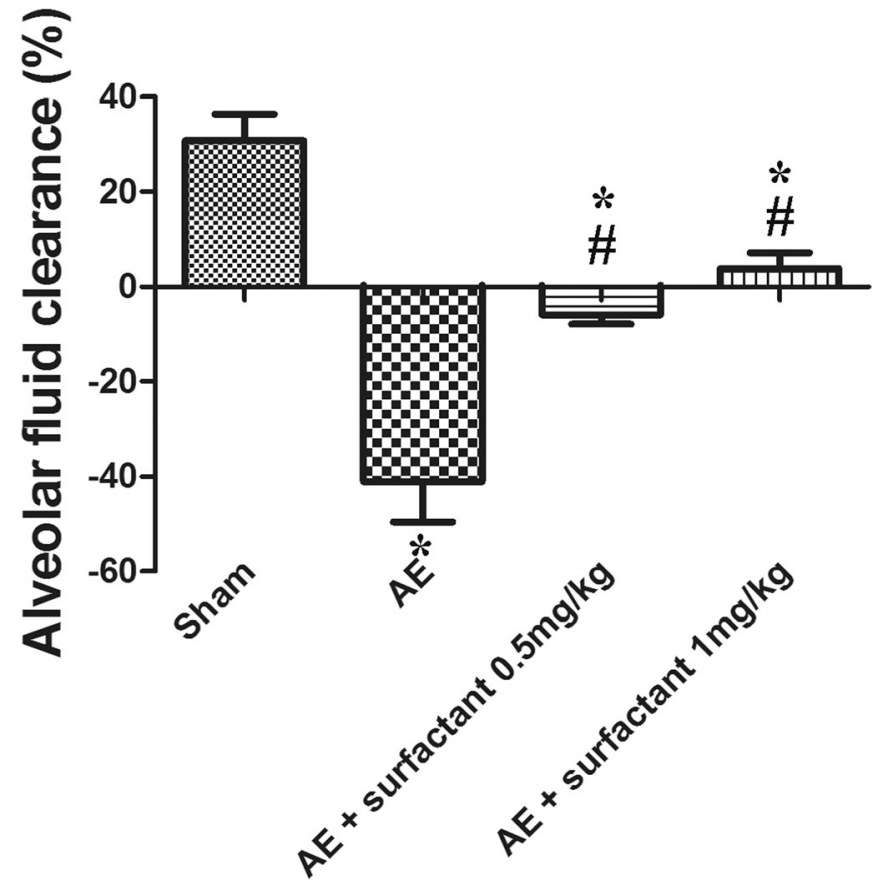

Fig. 3. Surfactant restored alveolar fluid clearance. Alveolar fluid clearance (AFC) decreased markedly in the air embolism (AE) group, compared to the sham group $(p<0.05)$. Surfactant $1 \mathrm{mg} / \mathrm{kg}$ significantly restored AFC, compared to the AE group $(p<0.05)$. There was a significant difference between the $*$ Sham $(p<0.05)$ and ${ }^{\#} \mathrm{AE}(p<0.05)$ groups.

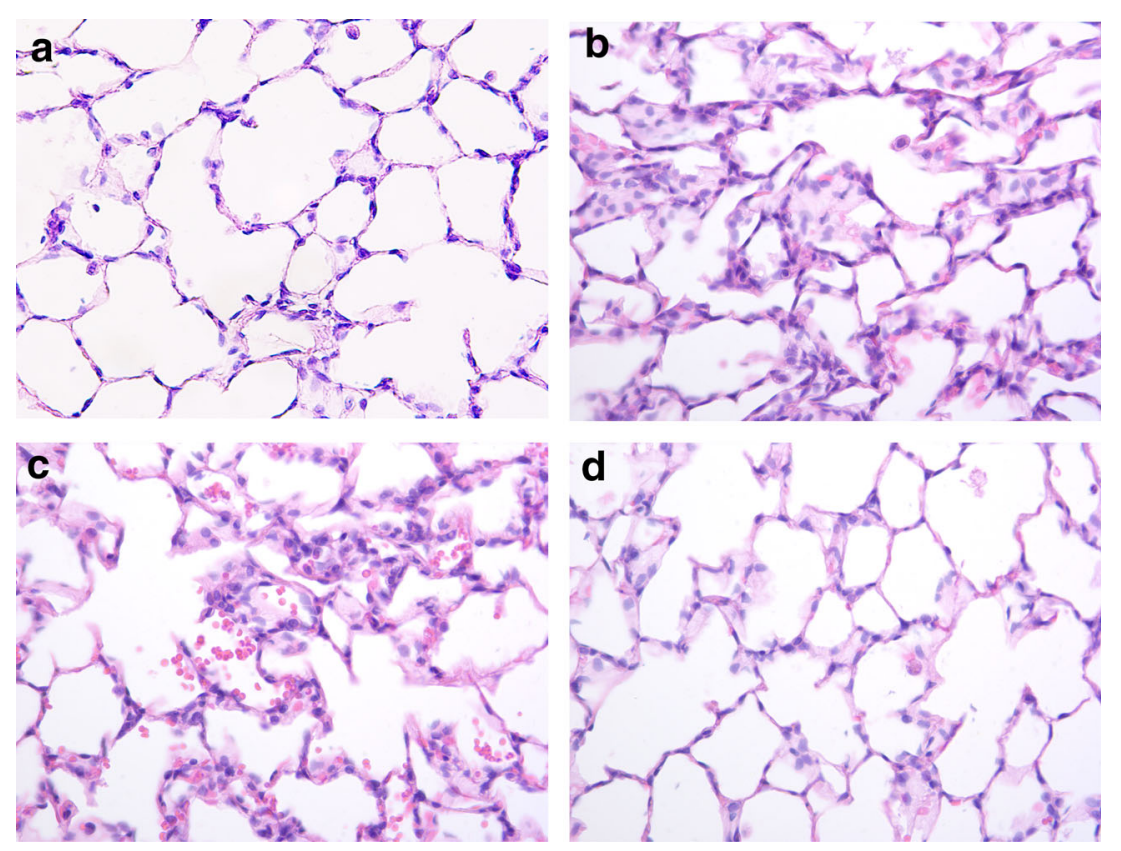

Fig. 4. Surfactant attenuated lung injury. a The rats in the sham group had normal histology, b while the air embolism (AE) group showed prominent neutrophilic sequestration and inter-alveolar septum thickening. $\mathbf{c}$ Lung injury was less severe in rat with surfactant $0.5 \mathrm{mg} / \mathrm{kg}$ and $\mathbf{d}$ was markedly attenuated by surfactant $1 \mathrm{mg} / \mathrm{kg}$. 


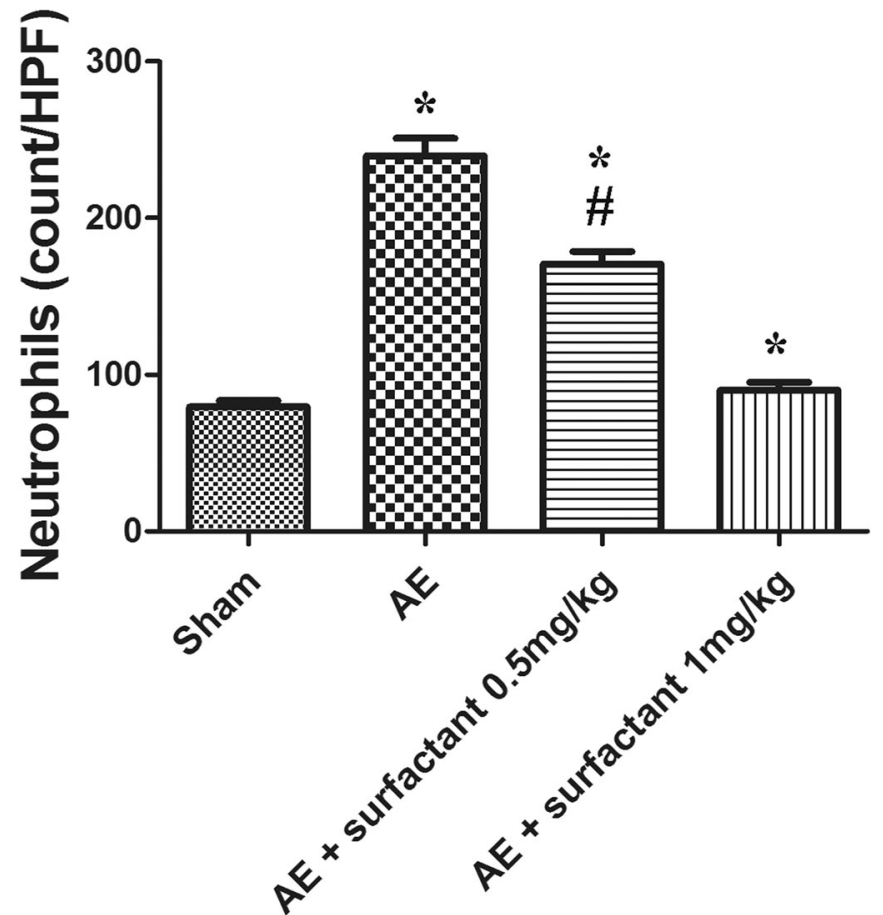

Fig. 5. Surfactant attenuated neutrophilic sequestration. Neutrophilic count in lung tissues was markedly increased in the air embolism (AE) group, compared to the sham group $(p<0.05)$, and significantly reduced by surfactant $1 \mathrm{mg} / \mathrm{kg}$, compared to the AE group $(p<0.05)$. There was a significant difference between the *Sham $(p<0.05)$ and ${ }^{\#} \mathrm{AE}(p<0.05)$ groups.

\section{DISCUSSION}

In the current study, AE-induced ALI showed pulmonary edema, pulmonary microvascular hyperpermeability, and lung inflammation with neutrophilic sequestration. AE-induced lung injury also presented with increased expressions of pro-inflammatory cytokines, activation of NF- $\mathrm{kB}$, and NKCC1. Surfactant administration attenuated AE-induced lung hyper-permeability, pulmonary edema, and lung inflammation with concurrent decreased expression of cytokines, NF-KB, and NKCC1. These results
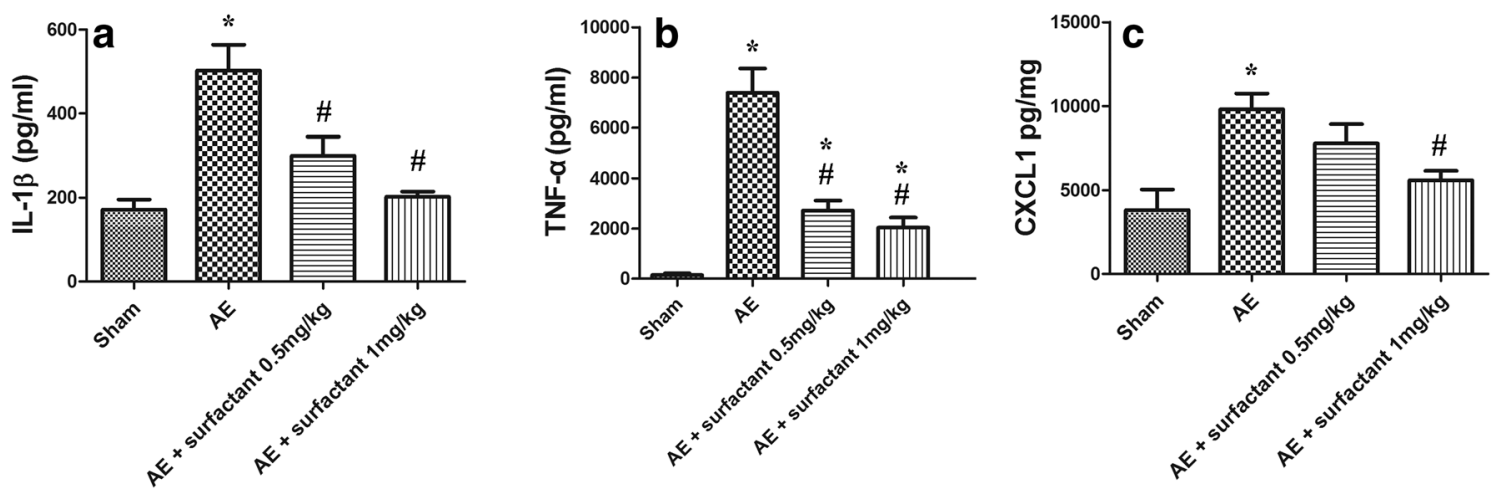

Fig. 6. Surfactant decreased expressions of pro-inflammatory cytokines. Expression levels of pro-inflammatory cytokines, a IL-1ß, b TNF- $\alpha$, and $\mathbf{c}$ CXCL-1 were significantly increased in the air embolism (AE) group, compared to the sham group $(p<0.05)$. Surfactant $1 \mathrm{mg} / \mathrm{kg}$ decreased expressions of these cytokines after $\mathrm{AE}(p<0.05)$. There was a significant difference between the $* \operatorname{Sham}(p<0.05)$ and ${ }^{\#} \mathrm{AE}(p<0.05)$ groups. Abbreviations: $\mathrm{AE}$, air embolism; IL-1ß: Interleukin-1 1 ; TNF- $\alpha$ : tumor necrosis factor- $\alpha$; CXCL-1: chemokine (C-X-C motif) ligand 1. 
a
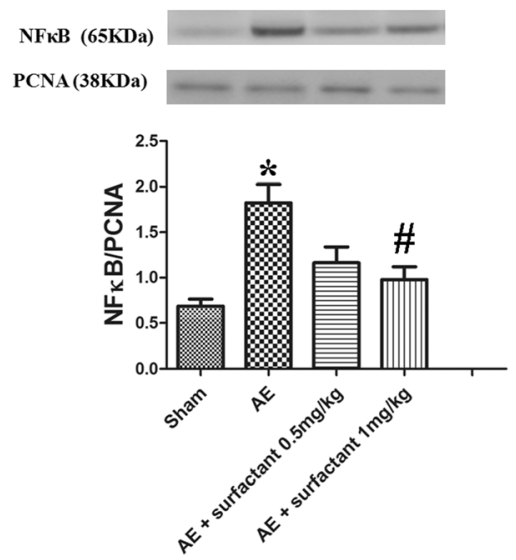

b

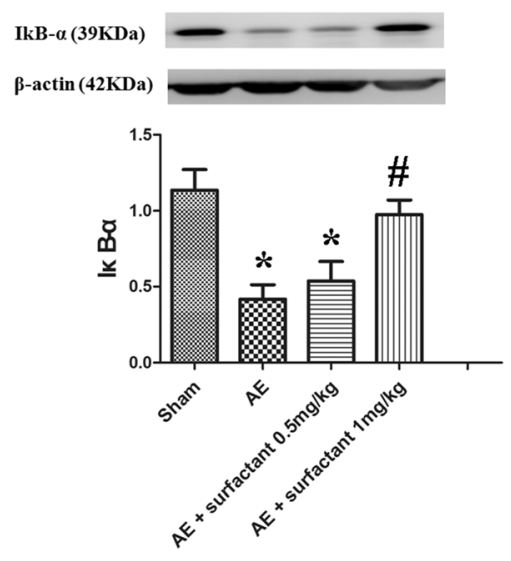

Fig. 7. Surfactant decreased air embolism-induced expression of NF-kB activation. In the air embolism (AE) group, the cytoplasmic level of phosphorylated NF-KB p65 a was increased, whereas I $\mathrm{kB}-\alpha \mathbf{b}$ was significantly suppressed compared to the sham group $(p<0.05)$. Surfactant $1 \mathrm{mg} / \mathrm{kg}$ reduced phosphorylated NF-KB p65 and restored IKB- $\alpha$ compared to the AE group $(p<0.05)$. There was a significant difference from the *Sham $(p<0.05)$ and ${ }^{\#} \mathrm{AE}(p<0.05)$ groups. Abbreviations: AE, air embolism; NF-kB, nuclear factor-kappa B; IкB- $\alpha$, inhibitor of NF-kB alpha.

suggest the therapeutic effects of surfactant in AE-induced ALI.

It is interesting to discuss the mechanisms of the protective effects of surfactant in AE-induced ALI.
Surfactant is important in the pathogenesis of ALI. Surfactant dysfunction contributes to the progressive loss of aerated volume, lung compliance, and worsening ventilation [6]. Surfactant aids in the reduction of alveolar surface
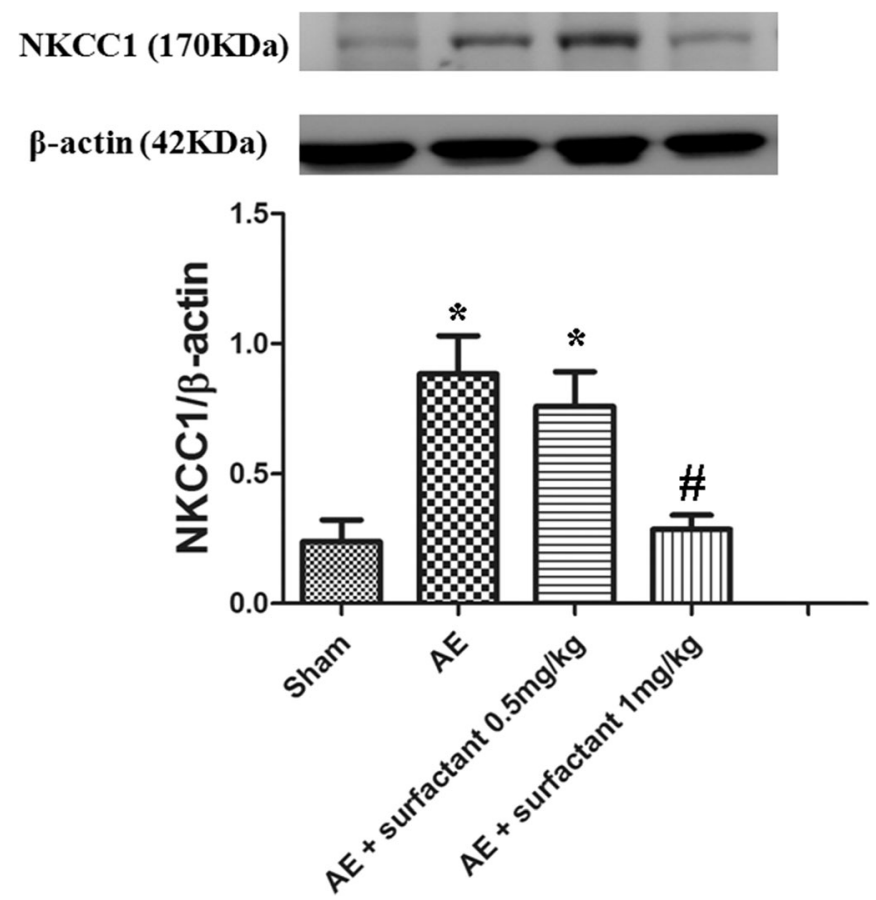

Fig. 8. Surfactant decreased air embolism-induced expression of NKCC1. The expression of NKCC1 was significantly increased in the air embolism (AE) group compared to the sham group $(p<0.05)$. Surfactant $1 \mathrm{mg} / \mathrm{kg}$ significantly decreased the expressions of NKCC1 compared to the AE group $(p<0.05)$. There was a significant difference between the *Sham $(p<0.05)$ and ${ }^{\#} \mathrm{AE}(p<0.05)$ groups. Abbreviations: AE, air embolism; Na-K-Cl cotransporter 1. 
tension and maintains alveolar opening and prevents alveolar collapse [20]. Hence, the surfactant can improve lung ventilation and attenuate the progression of lung injury [6]. We reveal that surfactant also protects against AE-induced lung injury.

Surfactant has immunomodulatory features with regulating pulmonary innate and acquired immunity and inflammatory processes [3]. Surfactant is known to decrease the release of pro-inflammatory cytokines and chemokines and further decrease lung inflammatory cells such as neutrophils, monocytes, and macrophages [3]. Therefore, therapeutic efficacy of the surfactant is related not only to its biophysical characteristics but also due to its antiinflammatory features [3]. In this study, we confirm that the surfactant decreases pro-inflammatory cytokines and neutrophils sequestration in AE-induced ALI.

$\mathrm{NF}-\kappa \mathrm{B}$ is a key transcription factor in cytokine gene expression and is important in ALI [11, 27]. Our results show activation of NF- $\mathrm{KB}$ pathway in AE-induced ALI. When cells are activated by inflammatory stimuli such as $\mathrm{AE}, \mathrm{I} \kappa \mathrm{B}$ is phosphorylated by IKK complex proteins [27]. Subsequently, NF- $\mathrm{kB}$ binds to $\mathrm{kB}$ enhancer elements of target genes to induce the transcription of inflammatory genes and increase expression of inflammatory cytokines [27]. The current study reveals the concurrent activation of NF- $\mathrm{KB}$ and pro-inflammatory cytokines after AE. After the administration of the surfactant, activation of NF- $\mathrm{KB}$ and pro-inflammatory cytokines was decreased.

Neutrophils play an essential role in the onset of ALI [5]. Activation of NF-KB pathway leads to increased expression of cytokines [27]. Pro-inflammatory cytokines have a chemotactic effect and lead to migration of neutrophils into the air spaces [27]. Migratory neutrophils further degranulate and release toxic intracellular products, free radicals, and cytotoxic enzymes that lead to lung injury [5, 8]. Surfactant decreases activation of NF-KB and proinflammatory cytokines, thereby decreases neutrophil sequestration in lungs and attenuating the severity of lung injury.

The regulation of surfactant in alveolar ion channels and fluid clearance is largely unknown. AFC was decreased in AE-induced lung injury and surfactant restored AFC after lung injury. Restoration of AFC represents a lung-protection mechanism via which excessive fluid is removed from the alveoli to restore ventilation for gas exchange [13]. Surfactant aids in the reduction of alveolar surface tension and prevents lung interstitial proteins and fluids leaking into the alveolar cavity [20]. Therefore, it is rational that surfactant can restore AFC in AE-induced lung injury.
NKCC1 is important in the regulation of lung water and inflammation. In previous studies, expression of NKCC1 was found to be increased in hyperoxia and ischemia-reperfusion-induced lung injury $[11,13]$. The animals with higher expression of NKCC1 showed more severe lung injury [10]. In this study, we also found increased expression of $\mathrm{NKCC} 1$ in AE-induced ALI. The activation of the IKK-NF- $\mathrm{KB}$ cascade causes osmotic stress and cell swelling, which can activate with-no-lysine kinase (WNK) kinases [21, 26]. Activation of WNK kinase has been shown to phosphorylate and activate NKCC1 [21].

$\mathrm{NKCC} 1$ influences both inflammatory responses and fluid regulation in the lungs $[13,16,24]$. The NKCC1 works to transport $\mathrm{Cl}^{-}$and is involved in the regulation of epithelial cell volume [17]. Up-regulating NKCC1 leads to dysregulation of fluid transport, cellular swelling, and inflammation $[16,18]$. NKCC1 regulates lung endothelial and epithelial barriers and modulates the inflammatory response to lung injury [13]. Therefore, increased expression of NKCC1 results in decreased alveolar liquid absorption, increased lung water, and inflammation. Conversely, inactivation of NKCC1 by surfactant leads to restored AFC, decreased lung water, and inflammation.

There are some limitations in our study. We suggest that the surfactant reduces the severity of AE-induced lung injury. This investigation provides a possible therapy in AE-induced lung injury in clinical settings. However, the present study has a few limitations. Firstly, the study was performed in animals using an isolated lung model. Further studies in human beings are warranted. Secondly, the present study is limited to the very early stage of AE-induced ALI. Further investigation is required to address the longer-term responses of surfactant in ALI.

\section{Clinical Implications}

The lungs are vulnerable solid organs. Pulmonary AE is a rare but potentially fatal complication of invasive medical or surgical procedures [7]. Therefore, it is important to study the effective treatments in AE-induced ALI. In the current study, we suggested that the surfactant could attenuate AE-induced ALI. This investigation provides incentive to explore impetus to consider approaches that address the role of surfactant within this the clinical setting.

\section{CONCLUSIONS}

AE-induced ALI presents with pulmonary edema, lung inflammation with neutrophilic sequestration, 
pulmonary microvascular hyper-permeability, and increased expressions of pro-inflammatory cytokines, NF$\kappa \mathrm{B}$, and NKCC1. Surfactant attenuates pulmonary edema, lung inflammation, and down-regulates the expression of pro-inflammatory cytokines, NF- $\mathrm{KB}$, and NKCC1. This study provides incentive to explore the effects of surfactant in the clinical setting. However, further investigation is still required to address the therapeutic effects of surfactant in ALI in humans.

\section{FUNDING INFORMATION}

This study was supported by grants from the Buddhist Tzu Chi General Hospital (TCRD TPE-104-33 and TCRD-TPE-105-25) and the Landseed Hospital (LS2015-03).

\section{COMPLIANCE WITH ETHICAL STANDARDS}

Competing Interest. The authors declare that they have no conflict of interest.

Open Access This article is licensed under a Creative Commons Attribution 4.0 International License, which permits use, sharing, adaptation, distribution and reproduction in any medium or format, as long as you give appropriate credit to the original author(s) and the source, provide a link to the Creative Commons licence, and indicate if changes were made. The images or other third party material in this article are included in the article's Creative Commons licence, unless indicated otherwise in a credit line to the material. If material is not included in the article's Creative Commons licence and your intended use is not permitted by statutory regulation or exceeds the permitted use, you will need to obtain permission directly from the copyright holder. To view a copy of this licence, visit http://creativecommons.org/licenses/by/4.0/.

\section{REFERENCES}

1. Alessi, D.R., J. Zhang, A. Khanna, T. Hochdörfer, Y. Shang, and K.T. Kahle. 2014. The WNK-SPAK/OSR1 pathway: Master regulator of cation-chloride cotransporters. Science Signaling 7 (334): re3.

2. Amigoni, A., A. Pettenazzo, V. Stritoni, and M. Circelli. 2017. Surfactants in acute respiratory distress syndrome in infants and children: Past, present and future. Clinical Drug Investigation 37: 729-736. https://doi.org/10.1007/s40261-017-0532-1.
3. Bersani, I., S. Kunzmann, and C.P. Speer. 2013. Immunomodulatory properties of surfactant preparations. Expert Review of AntiInfective Therapy 11 (1): 99-110.

4. Colin, J.M., B. Sasan, G.N. Sailendra, and O. Rahmi. 2017. Air embolism: diagnosis, clinical management and outcomes. Diagnostics 7 (1): 5 .

5. den Hengst, W.A., J.F. Gielis, J.Y. Lin, P.E. Van Schil, L.J. De Windt, and A.L. Moens. 2010. Lung ischemia-reperfusion injury: A molecular and clinical view on a complex pathophysiological process. American Journal of Physiology. Heart and Circulatory Physiology 299 (5): H1283-H1299.

6. Jin, F., and C. Li. 2017. Seawater-drowning-induced acute lung injury: From molecular mechanisms to potential treatments. Experimental and Therapeutic Medicine 13 (6): 2591-2598.

7. Kanchustambham, V., S. Saladi, K. Mehta, J. Mwangi, Z. Jamkhana, and S. Patolia. 2017. Vascular air embolism during bronchoscopy procedures- incidence, pathophysiology, diagnosis, management and outcomes. Cureus 9 (3): e1087.

8. Khimenko, P.L., G.J. Bagby, J. Fuseler, and A.E. Taylor. 1998. Tumor necrosis factor-alpha in ischemia and reperfusion injury in rat lungs. Journal of Applied Physiology 85 (6): 2005-2011.

9. Lan, C.C., C.K. Peng, S.F. Huang, K.L. Huang, and C.P. Wu. 2015. Activated protein $\mathrm{C}$ attenuates ischemia-reperfusion-induced acute lung injury. Experimental Lung Research 41 (5): 241-250.

10. Lan, C.C., C.K. Peng, S.E. Tang, H.J. Lin, S.S. Yang, C.P. Wu, and K.L. Huang. 2017. Inhibition of Na-K-Cl cotransporter isoform 1 reduces lung injury induced by ischemia-reperfusion. The Journal of Thoracic and Cardiovascular Surgery 153 (1): 206-215.

11. Lan, C.C., C.K. Peng, S.E. Tang, S.Y. Wu, K.L. Huang, and C.P. Wu. 2016. Anti-vascular endothelial growth factor antibody suppresses ERK and NF-KB activation in ischemia-reperfusion lung injury. PLoS One 11 (8): e0159922.

12. Li, M.H., K.L. Huang, S.Y. Wu, C.W. Chen, H.C. Yan, K. Hsu, C.W. Hsu, S.H. Tsai, and S.J. Chu. 2009. Baicalin attenuates air embolism-induced acute lung injury in rat isolated lungs. British Journal of Pharmacology 157 (2): 244-251.

13. Lin, H.J., C.P. Wu, C.K. Peng, S.H. Lin, S. Uchida, S.S. Yang, and K.L. Huang. 2015. With-no-lysine kinase 4 mediates alveolar fluid regulation in hyperoxia-induced lung injury. Critical Care Medicine 43 (10): e412-e419.

14. Muth, C.M., and E.S. Shank. 2000. Gas embolism. The New England Journal of Medicine 342 (7): 476-482.

15. Nakajima, D., M. Liu, A. Ohsumi, R. Kalaf, I. Iskender, M. Hsin, T. Kanou, M. Chen, B. Baer, R. Coutinho, L. Maahs, P. Behrens, S. Azad, T. Martinu, T.K. Waddell, J.F. Lewis, M. Post, R.A.W. Veldhuizen, M. Cypel, and S. Keshavjee. 2017. Lung lavage and surfactant replacement during ex vivo lung perfusion for treatment of gastric acid aspiration-induced donor lung injury. The Journal of Heart and Lung Transplantation 36 (5): 577-585.

16. Nguyen, M., A.J. Pace, and B.H. Koller. 2007. Mice lacking $\mathrm{NKCCl} 1$ are protected from development of bacteremia and hypothermic sepsis secondary to bacterial pneumonia. The Journal of Experimental Medicine 204 (6): 1383-1393.

17. O'Donnell, M.E., J.D. Brandt, and F.R. Curry. 1995. Na-K-Cl cotransport regulates intracellular volume and monolayer permeability of trabecular meshwork cells. The American Journal of Physiology 268 (4 Pt 1): C1067-C1074.

18. O'Donnell, M.E., L. Tran, T.I. Lam, X.B. Liu, and S.E. Anderson. 2004. Bumetanide inhibition of the blood-brain barrier $\mathrm{Na}-\mathrm{K}-\mathrm{Cl}$ cotransporter reduces edema formation in the rat middle cerebral artery occlusion model of stroke. Journal of Cerebral Blood Flow and Metabolism 24 (9): 1046-1056. 
19. Peng, C.K., K.L. Huang, C.P. Wu, M.H. Li, H.I. Lin, C.W. Hsu, S.H. Tsai, and S.J. Chu. 2010. The role of mild hypothermia in air embolism-induced acute lung injury. Anesthesia and Analgesia 110 (5): 1336-1342.

20. Puntorieri, V., J.Q. Hiansen, L.A. McCaig, L.J. Yao, R.A. Veldhuizen, and J.F. Lewis. 2013. The effects of exogenous surfactant administration on ventilation-induced inflammation in mouse models of lung injury. BMC Pulmonary Medicine 13: 67.

21. Richardson, C., and D.R. Alessi. 2008. The regulation of salt transport and blood pressure by the WNK-SPAK/OSR1 signalling pathway. Journal of Cell Science 121 (Pt 20): 3293-3304.

22. Souders, J.E. 2000. Pulmonary air embolism. Journal of Clinical Monitoring and Computing 16 (5-6): 375-383.

23. Wang, J., J.D. MacKenzie, R. Ramachandran, and D.Z. Chen. 2014. Identifying neutrophils in H\&E staining histology tissue images. Medical Image Computing and Computer-Assisted Intervention 17 (Pt1): 73-80.

24. Weidenfeld, S., and W.M. Kuebler. 2017. Cytokine-regulation of $\mathrm{Na}+\mathrm{K}+-\mathrm{Cl}$-cotransporter 1 and cystic fibrosis transmembrane conductance regulator-potential role in pulmonary inflammation and edema formation. Frontiers in Immunology 8: 393. https://doi.org/ 10.3389/fimmu.2017.00393.

25. Willson, D.F., and R.H. Notter. 2011. The future of exogenous surfactant therapy. Respiratory Care 56 (9): 1369-1386.

26. Yan, Y., and D. Merlin. 2008. Ste20-related proline/alanine-rich kinase: A novel regulator of intestinal inflammation. World Journal of Gastroenterology 14 (40): 6115-6121.

27. Yang, R., L. Yang, X. Shen, W. Cheng, B. Zhao, K.H. Ali, Z. Qian, and H. Ji. 2012. Suppression of NF-KB pathway by crocetin contributes to attenuation of lipopolysaccharide-induced acute lung injury in mice. European Journal of Pharmacology 674 (2-3): 391-396.

28. Yumoto, M., O. Nishida, F. Nakamura, and H. Katsuya. 2005. Propofol attenuates oxidant-induced acute lung injury in an isolated perfused rabbit-lung model. Journal of Anesthesia 19 (4): 287-294.

Publisher's Note Springer Nature remains neutral with regard to jurisdictional claims in published maps and institutional affiliations. 